\title{
TRATAMIENTO FARMACOLÓGICO DE LA INCONTINENCIA URINARIA POST PROSTATECTOMÍA RADICAL
}

\author{
Miguel Puyol y Argimiro Collado?.
}

Servicio de Urología. Hospital Sant Joan de Deu. Martorell Barcelona. Instituto Valenciano de Urología'. Valencia. España.

Resumen.- La incontinencia urinaria de esfuerzo (IUE) es un síntoma que se da en ambos sexos. En la IUE femenina el tratamiento farmacológico se ha demostrado eficaz pero el papel de este tratamiento en el varón es controvertido. Esta revisión evalúa los tipos de fármacos, sus efectos y los niveles de eficacia de estos estudios.

Palabras clave: Incontinencia urinaria en el Varón. Prostatectomía radical. Tratamiento farmacológico. Cáncer de próstata.
Summary.- Stress urinary incontinence (SUI) is a symptom appearing in both males and females. Pharmacological treatment has demonstrated to be effective for female SUI, but its role for treatment in males is controversial. This review evaluates the various types of drugs, their effects, and levels of efficacy in the studies.

Keywords: Male urinary incontinence. Radical prostatectomy. Pharmacological therapy. Prostate cancer.

\section{INTRODUCCIÓN}

La incontinencia urinaria (IU) definida como la manifestación por parte del paciente del escape de orina (1), es un síntoma común que afecta a ambos sexos. Aunque no es un proceso que ponga en peligro la vida del paciente, tiene un gran impacto en el bienestar psicosocial $(2,3)$ y en la calidad de vida (Qol) de los individuos que la padecen (4). La gran mayoría de los estudios epidemiológicos se ha realizado en mujeres, confirmando su elevada prevalencia, su impacto negativo sobre su calidad de vida y el gran número de recursos sanitarios que consume (5). La epidemiología de la IU en el varón, históricamente, no ha sido estudiada de la misma forma que en la mujer. Sin embargo, se han producido importantes progresos a partir de la última década del siglo pasado, particularmente gracias a estudios poblacionales de IU en el varón y más específicamente tras la prostatectomía radical.

Se han realizado varios estudios poblacionales para determinar la prevalencia de la IU en el 
varón. Publicándose tasas de prevalencia que oscilan entre un $3 \%$ y un $39 \%$ (6). Está amplia horquilla de resultados se explicaría bien por las diferencias en las poblaciones estudiadas, por la definición utilizada de incontinencia o por los métodos utilizados en las encuestas. Una estudio publicado en 2006, utilizando la última definición, 2002, de incontinencia urinaria de la ICS (International Continence Society), aporta una prevalencia de incontinencia para varones mayores de 60 años del 10,4\% y para menores de 60 años del $5,2 \%$ (7).

La prevalencia de la IU tras prostatectomía radical oscila entre el $2 \%$ hasta cerca del $60 \%(6)$. Estas amplias diferencias también se explicarían por las diferencias de las poblaciones estudiadas y por la definición utilizada. Las tasas de incontinencia recogidas de los síntomas notificados por los propios pacientes son generalmente 2 ó 3 veces mayores que las recogidas por los médicos. Estudios que han realizado ambas evaluaciones en la misma población confirman la observación que los médicos infravaloran la incontinencia post prostatectomía tanto como en un $75 \%(8,9)$. Las tasas de incontinencia parecen descender regularmente con el tiempo y estabilizarse en uno o dos años tras la cirugía (10). El déficit esfinteriano se relaciona generalmente con la IU tras prostatectomía radical, representando el 95\% del total de las causas. La hiperactividad del detrusor, definida como contracciones involuntarias con una amplitud mayor de $15 \mathrm{~cm} \mathrm{H}_{2} \mathrm{O}$, representa el 17\% (11).

La continencia urinaria es un mecanismo complejo, que precisa de la integración del sistema nervioso central y del periférico, así como de los músculos estriados y lisos.

Durante la fase de almacenamiento el detrusor debe relajarse para permitir la acomodación de un volumen creciente de orina a bajas presiones vesicales. La noradrenalina liberada por los nervios hipogástricos es el principal mediador del aumento de la acomodación vesical. Dentro de la vejiga la noradrenalina estimula principalmente los $\beta$-adrenoreceptores de las células musculares lisas, siendo principalmente del subtipo $\beta 3$ (12). Sin embargo, la relajación del detrusor $y$, por tanto, aumentar la acomodación vesical no es suficiente para obtener la continencia durante la fase de llenado. Adicionalmente, se requiere un aumento de la presión de cierre del cuello vesical, eso es, aumentar el tono del cuello vesical, uretra y musculatura pélvica. Con respecto a esto en el cuello vesical y en la uretra proximal el músculo liso es el principal generador de resistencia. En estos tejidos la noradrenalina liberada por los nervios hipogástricos estimula los receptores $\alpha 1$-adrenérgicos, principalmente del subtipo
alA (13), provocando la contracción. A diferencia a la uretra proximal, en la uretra distal predomina el músculo estriado, constituyendo el esfínter estriado o esfínter externo (14). Su acción se ve respaldada por los músculos estriados y ligamentos del suelo pélvico. Los nervios somáticos son los responsables de la contracción de los músculos estriados de la uretra y del suelo pélvico, por ejemplo del pudendo en la uretra y los nervios sacros en el suelo pélvico, a través de la acetilcolina.

Hasta ahora el "gold stantard" para el tratamiento de la IUE tras prostatectomía ha sido quirúrgico, en concreto la implantación de esfínter artificial (15). El objetivo del tratamiento farmacológico en estos casos es aumentar la resistencia de salida al flujo. Debido a que la incontinencia es un síntoma predominantemente femenino, hay poquísimos trabajos que estudien el tratamiento farmacológico en el varón. Además, se piensa que en las diferencias anatómicas entre el hombre y la mujer subyacen los diferentes mecanismos fisiopatológicos de la IUE. Incluso los efectos de drogas que actúan sobre las mismas estructuras del tracto urinario inferior en hombres y mujeres, pueden ser diferentes según el sexo. Por lo tanto, no podemos extrapolar a varones los datos del tratamiento farmacológico empleado en mujeres.

En esta revisión intentamos revisar la literatura con la intención de esclarecer la clase de fármacos empleados en el tratamiento del IUE en el varón.

\section{RESULTADOS}

En la literatura revisada encontramos trabajos utilizando agonistas $\alpha$-adrenérgicos, agonistas $\beta 2$-adrenérgicos o inhibidores de la recaptación de serotonina y noradrenalina potencialmente efectivos para el tratamiento de la IUE en el varón. La base de nuestra revisión son 8 trabajos que utilizan fármacos de estas tres categorías farmacológicas.

\section{Agonistas de los receptores $\alpha$-adrenérgicos}

Al aumentar la resistencia de salida vesical durante la fase de llenado los agonistas $\alpha$-adrenérgicos se han utilizado para el tratamiento de la IUE (16).

Hay muy pocos trabajos publicados sobre el papel de estos fármacos en el varón. En una reciente publicación (17), 2007, sobre el uso de estos agentes, se revisan 22 estudios con distribución aleatoria, ninguno de ellos incluía varones, concluyen que estos fármacos son mejores que el placebo en cuanto a la reducción del número de pañales utilizados y en el 
número de episodios de incontinencia, así como, en una mejoría subjetiva de los síntomas.

Nosotros hemos encontrado dos trabajos, de series de casos, ambos publicados en la década de los setenta del siglo pasado. El primero (18) utiliza efedrina (44-200 mg, cuatro veces al día) y el segundo (19) fenilpropanolamina (4 mg al día). Ambos estudios incluyen tanto hombres como mujeres, la etiología es heterogénea y sólo evalúan resultados clínicos. Estos fármacos no son selectivos para los receptores uretrales y pueden causar efectos sistémicos severos como hipertensión, trastornos del sueño, nauseas, sequedad de boca, cefalea, temblores, palpitaciones y empeoramiento de ritmos cardiacos anormales (20). Kerman publica que mujeres menores de 50 años, que han estado tomando fenilpropanolamina como anorexígeno, presentan un riesgo 16 veces mayor de padecer accidentes cerebrovasculares hemorrágicos que mujeres de su misma edad que no hayan tomado nunca este fármaco (21). En Noviembre del 2000 la FDA (Food and Drug Administration) retiro del mercado todos los productos con fenilpropanolamina por el riesgo de padecer accidentes cerebrovasculares hemorrágicos (22). En España no existe ninguna especialidad farmacéutica que contenga fenilpropanolamina para la indicación de supresión del apetito (23).

Para disminuir los efectos secundarios, tendríamos que disponer de fármacos más uroselectivos, es decir, que actuaran sobre los receptores $\alpha$-1A-adrenérgicos, los cuales parecen ser los responsables de la contracción del músculo liso uretral (24). Musselman (25) publica un estudio randomizado y cruzado fase 2 con Ro 115-1240, un agonista parcial selectivo del receptor adrenérgico $\alpha$-IA/IL (26), en 37 mujeres afectas de IUE de leve a moderada. Demostró un efecto positivo, en la reducción del número de episodios de incontinencia y del número de pañales utilizados. Pero los efectos secundarios reducen la utilización futura de este fármaco.

\section{Agonistas de los receptores $\beta 2$-adrenérgicos.}

Generalmente se reconoce que el estímulo de los receptores adrenérgicos disminuye la presión uretral (27). Pero también se ha publicado que el estímulo de los receptores $\beta_{2}$-adrenérgicos aumenta la contracción de algunas fibras rápidas del músculo estriado y suprime la contracción de algunas fibras de contracción lenta $(24,28)$.

Kishimoto y cols. demuestran que, utilizando preparaciones de músculo estriado periuretral de conejo, el clembuterol (Figura 1), un agonista de los receptores $\beta_{2}$-adrenérgicos, produce una contracción de dichas fibras. Una acción que es antagonizada por el propanolol. Estos datos sugieren que la respuesta al clembuterol está mediada por receptores $\beta$-adrenérgicos. También explica que el aumento de la presión uretral mediado por el clembuterol puede ser atribuidos a un aumento de la tensión del músculo estriado periuretral. Estos autores son los primeros en publicar el aumento de la presión uretral producida por el clembuterol y especularon su potencial uso en el tratamiento de la IUE (29).

Hemos encontrado dos estudios de pacientes afectos de IU tratados con clembuterol.

En el primero, Noguchi y cols (30) tratan a 14 varones con IU tras PR, administraron $20 \mathrm{mg}$ de clembuterol dos veces al día durante un mes, con evaluación urodinámica antes y después del tratamiento médico. Al final del tratamiento, 9 pacientes (64\%) presentaron una mejoría en el número de pañales con un aumento significativa de la longitud funcional uretral. En este artículo no menciona el tipo de incontinencia que presentan los pacientes.

En una segunda publicación, se tratan con $20 \mathrm{mg}$ de clembuterol, tres veces al día, a 72 varones con IU tras resección transuretral de próstata por hipertrofia benigna de próstata. Un 76,3\% de los varones refirió una mejoría de su IU (31). Los autores no definen que es para ellos curación o mejoría. Los efectos adversos del clembuterol son temblores, taquicardias, arritmias, sudoraciones profusas y cefaleas. Como este fármaco no ha sido testado en ensayos clínicos rigurosos, no ha recibido por parte de la FDA ni de la European Agency for the Evaluation of Medical Productos (EMEA) la autorización para poderse aplicar en esta patología.

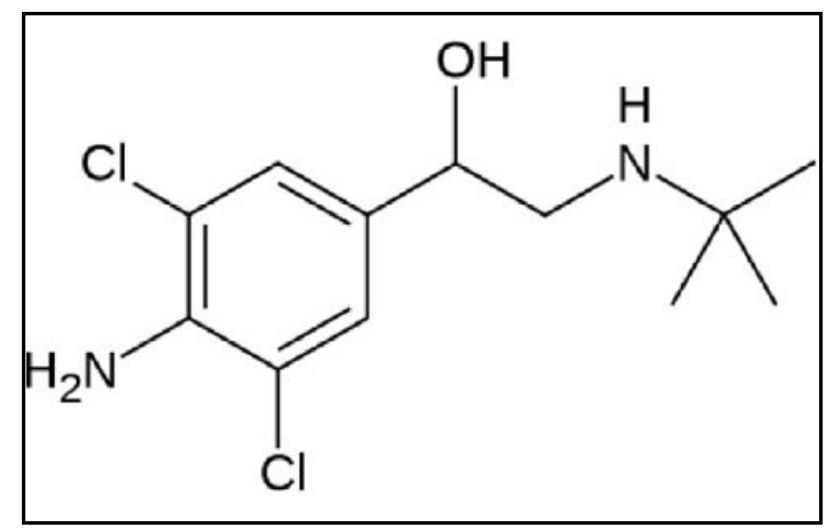

FIGURA 1. Estructura química del clembuterol. 


\section{Inhibidores de la recaptación de serotonina y nora- drenalina.}

\section{a). Imipramina.}

La imipramina, un antidepresivo tricíclico. Su mecanismo de acción radica en su capacidad de inhibir la recaptación de noradrenalina (NE) y serotonina (5-HT), aumentando la disponibilidad de estos neurotransmisores en el sistema nervioso central. Fue el primer inhibidor de la recaptación de 5-HTy NE investigado para el tratamiento de la IUE masculina (32). En este estudio, 19 pacientes con IU tras prostatectomía por hiperplasia benigna de próstata, se dividieron en tres brazos, el primero fue tratado con imipramina, el segundo con Eskornade (una mezcla de tres fármacos, un anticolinérgico la isopropamida, un agonista $\alpha$-adrenérgico la fenilpropanolamina y un antihistamínico la difenilpiralina) y el tercer brazo con emepronium, un anticolinérgico. En el primer grupo se incluyeron 5 pacientes, 12 en el segundo brazo y dos en el tercero. En el primer grupo tres pacientes estaban secos $(60 \%)$, en el segundo solo uno $(8 \%)$ y ninguno en el tercer grupo. No se mencionan ni las dosis y el tiempo transcurrido tras la cirugía. Después de esta inicial publicación no se ha publicado ningún estudio con este fármaco para el tratamiento de la IUE masculina.

\section{b). Duloxetina.}

La duloxetina (Figura 2) es un inhibidor dual de la recaptación de 5-HT y NE. La EMEA lo aprobó en Agosto del 2004 para el tratamiento de la IUE en la mujer (33), en España también está autorizada para el tratamiento de la incontinencia urinaria femenina por estrés moderada o grave (34).

La duloxetina, en un modelo de gatos anestesiados, aumenta significativamente la capacidad vesical y la actividad esfinteriana, por una acción sobre el SNC mediada por vía somática y por una modulación sensorial aferente. Favorece el almacenamiento vesical por la relajación del detrusor y por el aumento de la resistencia uretral (35). Estos efectos no se han reproducido tras la administración por separado de dos simples inhibidores de la recaptación de 5-HT o NE (36).

Su acción, posiblemente, la obtiene bloqueando la recaptación de 5-HT y NE en el núcleo de Onuf, un área localizada en la médula sacra que posee una gran densidad de receptores de 5-HT y NE. El bloqueo de la recaptación de 5-HT y NE aumenta la activación de receptores $\alpha_{1}$-adrenérgicos y serotoninérgicos $\left(5 \mathrm{HT}_{2}\right)$, facilitando la acción del glutamato, con lo que aumenta la actividad del nervio pudendo, aumentando por ende el tono del esfínter uretral externo (35-37).
Considerando estos efectos en el esfínter estriado de la mujer, es creíble esperar que tales efectos se reproduzcan en el varón, siempre que la estructura nerviosa del esfínter estriado no esté totalmente dañada. Aunque la duloxetina presenta una eficacia, seguridad y tolerabilidad similares entre hombres y mujeres afectos de trastornos depresivos mayores (38), esto no significa que los efectos sobre la continencia urinaria sean los mismos.

Los datos de los efectos de la duloxetina en varones derivan de tres estudios publicados entre 2006 y 2007 (39-41). Schlenker y cols. presentan los resultados preliminares de un estudio utilizando duloxetina, de forma compasiva (off-label), en varones con IUE a las 3 o más semanas después de la retirada de la sonda tras prostatectomía radical $(\mathrm{N}=15)$ o cistoprostatectomía radical con neovejiga ortotópica ileal $(\mathrm{N}=5)$ (39). Definen la incontinencia como el uso de tres o más pañales al día a pesar de ejercicios intensos de rehabilitación del suelo pélvico. Dieciocho de los 20 pacientes tomaron duloxetina regularmente, a una dosis de $40 \mathrm{mg}$ dos veces al día. El número medio de pañales día antes del tratamiento era de 8 , tras un promedio de tratamiento de 9,4 semanas el número medio de pañales día desciende a 4,2 ( $P<0,001)$. Quince de los 18 varones $(83,33 \%)$ refieren una mejoría de la IUE tras el uso del fármaco y $7(35 \%)$ están completamente secos o utilizan un solo pañal al menos por razones de seguridad.

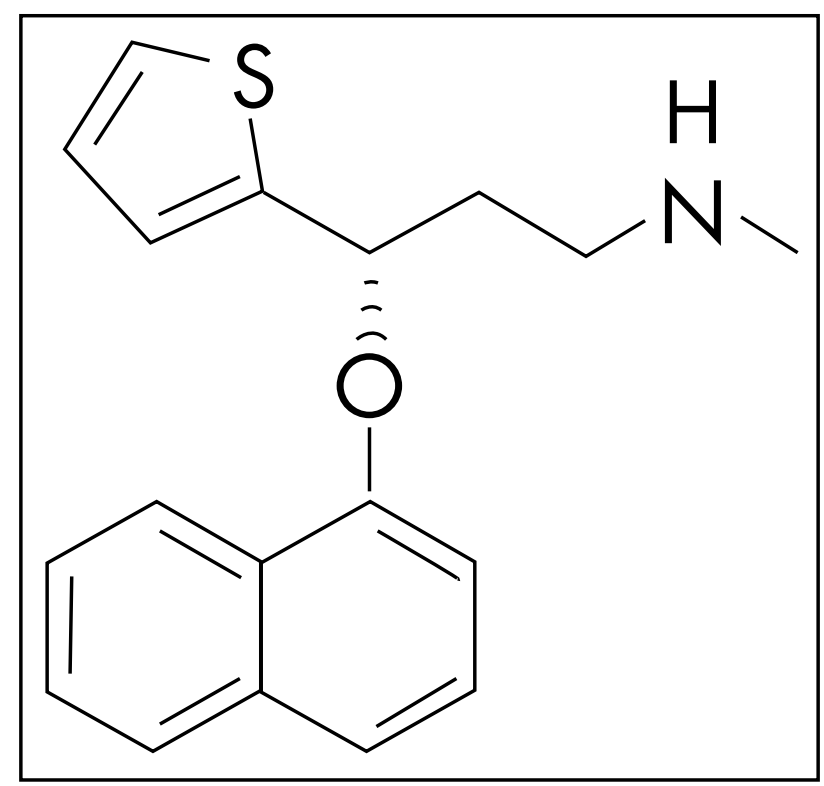

FIGURA 2. Estructura química de la duloxetina. 
También notifican una alta tasa de efectos secundarios en estos varones. Catorce de los 20 pacientes refieren efectos secundarios, y estos efectos secundarios son los responsables de que dos pacientes no entren en el ensayo por miedo a sus efectos adversos. Seis $(30 \%)$ discontinúan por efectos secundarios. Los seis pacientes restantes presentan efectos secundarios leves, como fatiga, sequedad de boca, nauseas o insomnio que desaparecen dentro de las cuatro primeras semanas. Ciertamente, como en las mujeres tratadas con duloxetina, la tolerabilidad al fármaco es un asunto importante en el uso de este medicamento. Los pacientes que continúan con el fármaco lo hacen en un promedio de 9,4 semanas, y no está claro que les pasó después y por que dejaron de tomar el fármaco.

En otro estudio, Zahariou y cols. evalúa la eficacia de la duloxetina en 18 pacientes que refieren IUE al año de la prostatectomía radical y su impacto en parámetros urodinámicos tales como la presión abdominal del punto de fuga (ALPP), presión de cierre uretral máxima (MUCP) y la presión retrógrada de punto de fuga (RLPP) (40). Inicialmente todos los pacientes recibieron $20 \mathrm{mg}$ de duloxetina dos veces al día para evitar los efectos secundarios. Tras la primera semana, la dosis se aumenta a $40 \mathrm{mg}$ dos veces al día. Se utilizó el test del pañal (pad test) de una hora para verificar y cuantificar la incontinencia urinaria. Ocho pacientes usaban 2 pañales $(44,5 \%)$, 8 pacientes precisaban tres pañales $(44,5 \%)$ y 2 (11\%) usaban más de tres pañales al día. La pérdida urinaria oscilaba entre 90 y 210 gramos a la hora (media 124 gramos).

A los tres meses de tratamiento, doce pacientes utilizaban 2 pañales al día $(66,6)$ y seis utilizaban 3 pañales al día $(33,3 \%)$. La pérdida medida por el test del pañal disminuyó a 34-178 gramos a la hora (media 58 gramos), siendo estadísticamente significativa $(\mathrm{P}<0,001)$. Sólo la $M U C P$, de todos los parámetros urodinámicos evaluados, mejoro significativamente $\left(52,5 \pm 19,4 \mathrm{cmH}_{2} \mathrm{O}\right.$ a $67,3 \pm 17,3$ $\mathrm{cmH}_{2} \mathrm{O}$ ) probablemente debido a la influencia de la duloxetina sobre el esfínter extrínseco. En este trabajo no se habla para nada de los efectos secundarios y tampoco de lo que pasa después de dejar el tratamiento.

Filocamo y cols. publican un estudio, prospectivo, aleatorio y doble ciego, cuyo objetivo fue evaluar la eficacia y la seguridad de la asociación de duloxetina $40 \mathrm{mg}$ dos veces al día y rehabilitación (Grupo A) frente a rehabilitación y placebo (Grupo B) en varones con IUE tras prostatectomía radical, y comparar la tasa de continencia después de la finalización planificada del estudio (41).
Diez pacientes discontinúan el estudio prematuramente por efectos adversos, nueve $(15,2 \%)$ en el grupo $A$ y $1(1,8 \%)$ en el grupo $B(P=0,01)$, siendo las nauseas $(70 \%)$ la primera causa del abandono. Ciento dos pacientes finalizan las 24 semanas del estudio, 50 en el grupo A y 52 en el B. Ambos grupos presentan mejoría significativa, incluso tras las primeras semanas del estudio $(P<0,001)$. Tras cuatro meses de tratamiento, 34 pacientes $(57,6 \%)$ en el grupo $A$ y $27(51,9 \%)$ en el grupo $B$ son continentes $(P=0,007)$. A las 20 semanas, cuatro semanas tras finalizar el tratamiento con el fármaco y continuar sólo con ejercicios de rehabilitación, se observa una vuelta inversa de los resultados, $23(46 \%)$ pacientes del grupo A y $38(73 \%)$ del B son continentes, están completamente secos. Sin embargo, a las 24 semanas, $31(62 \%)$ en el grupo $A$ versus 41 (78\%) están secos.

Los autores conjeturan sobre este fenómeno, achacándolo a que los pacientes del grupo $A$ están menos motivados y por esto menos dispuestos a realizar unos ejercicios correctos de rehabilitación comparados con los del grupo placebo.

Finalmente haremos referencia a nuestros resultados con duloxetina. El estudio la comprenden 56 pacientes con IUE al año de la prostatectomía radical, incontinencia establecida. Utilizamos la duloxetina, como uso compasivo, a $30 \mathrm{mg}$ dos veces al día durante 9 meses.

Diecisiete pacientes $(37 \%)$ abandonan precozmente el medicamento por efectos adversos y 20 $(43 \%)$ por falta de eficacia. Sólo 9 pacientes (20\%) finalizan el tratamiento. La continencia se evaluó mediante el número de pañales/ día y cuestionario ICIQ-IU SF, validado al español. Al inicio del tratamiento la media de pañales/día era de 1,8 y la puntuación del cuestionario de 12,5 . A las 12 semanas de tratamiento el $34 \%$ de las pacientes no utilizan pañales, el número medio de pañales/día es 1,2 y la puntuación del cuestionario ICIQ-IU SF es de 9,39. A los nueve meses de tratamiento, la media de pañales/día descendió a 0,6 y la puntuación media del cuestionario a 6,3 , y el $63 \%$ de los pacientes no utilizan pañales. De los nueve pacientes que finalizan el tratamiento, en siete se mantiene la continencia y en los dos restantes empeora. Los efectos secundarios más frecuentes fueron el temblor fino en extremidades y el mal estar general. Nosotros dábamos el fármaco tras las comidas para disminuir las náuseas, curiosamente algunos pacientes presentaron somnolencia.

Nuestras conclusiones son que la duloxetina tiene un efecto moderado en la incontinencia "establecida" (>1 año tras PTR) que puede plantearse pre- 
viamente a la cirugía, debido a que sus potenciales efectos beneficiosos se constatan ya en la primera visita pero hay que advertir de las tasas de éxito y de los efectos secundarios. Las pegas a este estudio puede ser que no es un estudio controlado con placebo, aunque en nuestra descarga un gran porcentaje había sido tratado con anti-colinérgicos sin éxito. Lo segundo, es el porcentaje menor de utilización de compresas, ya que los pacientes que utilizan más de 3 compresas al día son orientados a la implantación de un esfínter urinario artificial.

\section{CONCLUSIONES}

Aunque principalmente se ha estudiado el tratamiento médico de la IUE en mujeres, algunos estudios se han realizado en varones con IUE, especialmente después de prostatectomía radical. Muchos de estos estudios adolecen de un apropiado diseño para evaluar los efectos del tratamiento y tienen un bajo nivel de evidencia (nivel 4 de la clasificación de Oxford (43)). La duloxetina un fármaco con una eficacia probada en la IUE femenina parece ser efectiva también en varones. Aunque no disponemos de muchos estudios con este fármaco, hemos encontrado tres publicados y uno presentado en un congreso, sólo uno es aleatorio y controlado con placebo (nivel $1 \mathrm{~b}$ de la clasificación de Oxford, (43)) en el cual la duloxetina se combina con ejercicios de rehabilitación del suelo pélvico, las evidencias sugieren que este fármaco puede tener un lugar en el manejo de varones con IUE. Necesitamos disponer de estudios mayores y bien diseñados que confirmen los resultados previos y poder recomendarla para un uso rutinario.

\section{BIBLIOGRAFÍA y LECTURAS RECOMENDADAS (*lectura de interés $y^{* *}$ lectura fundamental)}

1. Grupo Español de Urodinámica y de SINUG. Consenso sobre terminología y conceptos de la función del tracto urinario inferior. Actas Urol Esp, 2005; 29 (1): 16-30

2. Hunskaar S, Sandvik H. One hundred and fifty men with urinary incontinence. III Psychosocial consequences. Scand J Prim Health Care, 1993; 11: 193-196.

3. Shaw C. A review of the psychosocial predictors of helpseeking behaviour and impact on quality of life in people con urinary incontinence. J. Clin Nurs, 2001; 10: 15-24.

4. Herzog AR. Fultz AH. Brock BM. Brown MB. Diokno AC. Urinary incontinence and pysochological distress among older adults. Psychol Aging, 1988; 3: 115-121.
5. Hunskaar S, Burgio K, Diokno AC, Herzog AR, Hjälmas K, Lapitan MC. In: Abrams P, Cardozo L, Khoury S, Wein A, Editors. Incontinence: 2nd International Consultation on Incontinence. 2nd ed. Plymouth, UK: Health Publications Ltd; 2000. P. 165-2001.

6. Hunskaar S, Burgio K, Clark, A, Lapitan MC, Nelson R, Sillen U, Thom,D. In: Abrams P, Cardozo L, Khoury S, Wein A, Editors. Incontinence: 3nd International Consultation on Incontinence. 3nd ed. 2005. P. 225-312.

*7. D. Irwin I Milsom, S Hunskaar, K Reilly, Z Kopp, S Herschorn, K Coyne, C Kelleher, C Hampel, W Artibani, P Abrams. Population-Based Survey of Urinary Incontinence, Overactive Bladder, and Other Lower Urinary Tract Symptoms in Five Countries: Results of the EPIC Study. Eur Urol, 2006, 50: 1306-1315.

*8. Mc Cammon K, Kolm P, Main B and Schellhammer P. Comparative quality-of-life analysis after radical prostatectomy or external beam radiation for localized prostate cancer. Urology, 1999, 54: 509-516.

*9. Olsson L, Salomon A, Nadu, HoznekA, Cicco A, Saint F, Chopin D, and Abbou C. Prospective patient-reported continence after laparoscopic radical prostatectomy. Urology, 2001; 58: 570-572.

10. Walsh P, Marschke P, Ricker D, and Burnett A. Patient-reported urinary continence and sexual function after anatomic radical prostatectomy. Urology, 2000, 55: 58-61.

11. Kielb $\mathrm{S}$ and Clemens Q. Comprehensive urodynamics evaluation of 146 men with incontinence after radical prostatectomy Urology, 2005; 55: 2-4.

12. Yamaguchi O. $\alpha 3$-Adrenoceptors in human detrusor muscle. Urology, 2002; 59(Suppl 5A):25-29.

13. Nasu K, Moriyama N, Fukasawa R, Tsujimoto G, Tanaka T, Yano J and Kawabe K. Quantification and distribution of a1-adrenoceptor subtype $\mathrm{mR}$ NAs in human proximal urethra. Br J Pharmacol, 1998;123: 1289-1293.

14. Bannowsky A, Juenemann K P. Innervation and function of the female urinary bladder and urethra. EAU Update Series, 2003; 1:120-17.

15. Montague DK, Angermeier KV. Postprostatectomy urinary incontinence: the case for artificial urinary sphincter implantation. Urology, 2000; 55: 2-4.

16. Brune ME, O'Neill AB, Gauvin DM, Katwala SP, Altenbach RJ, Brioni JD, Hancock AA, Sullivan JP. Comparison of alpha 1-adrenoceptor agonists in canine urethral pressure profilometry and abdominal leak point pressure models. J Urol, 2001;166(4):1555-9

17. Alhasso AA, Glazener CMA, Pickard R, N'Dow JMO. Adrenergic drugs for urinary incontinence in adults (Review). The Cochrane Library 2008, Issue 4 http://www.thecochranelibrary.com. 
**18. Diokno A, Taub M. Ephedrine in treatment of urinary incontinence. Urology, 1975; 5: 624-625.

**19. Awad SA, Downie JW, Kiruluta HG. Alpha-adrenergic agents in urinary disorders of the proximal urethra. Part I. Sphincteric incontinence. Br. J. Urol, 1978; 50(5):332-5.

20. Andersson K, Appell R, Cardozo L, Chapple C, Drutz H, Fourcroy J, Nishizawa O, Vela Navarrete R an Wein A. In: Abrams P, Cardozo L, Khoury $\mathrm{S}$, Wein A, Editors. Incontinence: $3^{\text {nd }}$ International Consultation on Incontinence. $3^{\text {nd }}$ ed. 2005. P. 809-854.

21. Kernan W, Viscoli C, Brass L, Broderick J, Brott T, Feldmann E, Morgenstern L, Wilterdink J and Horwitz R. Phenylpropanolamine and the risk of hemorrhagic stroke. Engl J Med, 2000; 343:1826-32.

22. Fleming G. The FDA regulation and the risk of stroke. N Engl J Med, 2000; 343:1886-7.

23. Agencia española de medicamentos y productos sanitarios. Actividad. Alertas. Uso humano. http://www.agemed.es/actividad/alertas/usoHumano/seguridad/fenilpropanolamina.htm. Último acceso Diciembre del 2008.

24. Michel M and Vrydag W. $\alpha 1-, \alpha 2$ - and $\beta$-adrenoceptors in the urinary bladder, urethra and prostate. Br. J. Pharmacol 2006; 147 (Suppl 2): S88 -s119.

25. Musselman DM, Ford AP, Gennevois DJ, 1. A randomized crossover study to evaluate Ro 115-1240, a selective alpha1 A/1L-adrenoceptor partial agonist in women with stress urinary incontinence. BJU Int, 2004;93:78-83.

26. Blue DR, Daniels DV, Gever JR, Jett MF, O'Yang C, Tang HM, et al. Pharmacological characteristics of Ro 115-1240, a selective $\alpha-1 \mathrm{~A} / 1 \mathrm{~L}$-adrenoceptor partial agonist: a potential therapy for stress urinary incontinence.

27. Andersson K and Wein A. Pharmacology of the Lower Urinary Tract: Basis for Current and Future Treatments of Urinary Incontinence. Pharmacol Rev 56:581-631, 2004

28. Cairns S. and Dulhunty A. The effects of _-adrenoceptor activation on contraction in isolated fastand slow-twitch skeletal muscle fibres of the rat. Br. J. Pharmacol,1993; 110, 1133-1141.

29. Kishimoto T, Morita T, Okamiyak Y, Hoshina E, and Takeshita T. Effect of clembuterol on contractile response in periurethral striated muscle of rabbits. Tohoku J. Exp. Med, 1991; 165, 243-245

**30. Noguchi M, Eguchi Y, Ichiki J, Yahara J, Noda S. Therapeutic efficacy of clenbuterol for urinary incontinence after radical prostatectomy. Int $\mathrm{J}$ Urol, 1997; 4(5):480-3.

**31. Zozikov B, Kunchev SI, Varlev C. Application of clenbuterol in the treatment of urinary incontinence. Int Urol Nephrol, 2001; 33, (3), pp. 413-416.

**32. Reid GF, Fitzpatrick JM, Worth PH. The treatment of patients with urinary incontinence after prostatectomy. Br J Urol, 1980; 52(6):532-4.

33. The European Agency for the Evaluation of Medical Products. Evaluation of medicines for human use. http://www.emea.europa.eu/pdfs/human/ press/pr/123804en.pdf. Último acceso Diciembre del 2008.

*34. Agencia española de medicamentos y productos sanitarios. Prof. Salud humana. Guía de prescripción terapéutica. http://www.imedicinas.com/GPTage. Último acceso Diciembre del 2008.

*35. Thor KB and Katofiasc MA. Effects of duloxetina, a combined serotonin and norepinephrine reuptake inhibitor, on central neural contrl of lower urinary tract function in the chloralose anesthetized female cat. J. Pharmacol Exp Ther, 1995; 274 (2): 1014-1024.

36. Katofiasc MA, Nissen J, Audia JE, Thor KB. Comparison of the effects of serotonin selective, norepinephrine selective, and dual serotonin and norepinephrine reuptake inhibitors on lower urinary tract function in cats. Life Sci. 2002; 71(11):1227-36.

*37. Thor KB. Serotonin and norepinephrine involvement in efferent pathways to the urethral rhabdosphincter: Implications for treating stress urinary incontinence. Urology 2003; 62 (Suppl 4 A): 3-9.

38. Kornstein SG, Wohlreich MM, Mallinckrodt $\mathrm{CH}$, Watkin JG, Stewart DE. Duloxetine efficacy for major depressive disorder in male vs. female patients: data from 7 randomized, double-blind, placebo-controlled trials. J.Clin Psychiatry 2006; 67: 761-770.

**39. Schlenker B, Gratzke C, Reich O, Schorsch I, Seitz M, Stief CG. Preliminary results on the offlabel use of duloxetine for the treatment of stress incontinence after radical prostatectomy or cystectomy. Eur Urol, 2006; 49: 1075-1078.

**40. Zahariou A, Papaioannou P, Kalogirou G. Is $\mathrm{HCl}$ duloxetine effective in the management of urinary stress incontinence after radical prostatectomy? Urol Int, 2006; 77(1):9-12.

**41. Filocamo MT, Li Marzi V, Del Popolo G, Cecconi F, Villari D, Marzocco M, Nicita G. Pharmacologic treatment in postprostatectomy stress urinary incontinence. Eur Urol, 2007; 51: 1559-1564.

42. Collado A, Rubio J, Iborra I, Puyol M, Casanova J, Solsona E. Tratamiento con duloxetina de la incontinencia de esfuerzo "establecida" tras prostatectomía radicalLXXXIII Congreso Nacional de Urología. Barcelona 2008. Póster 234. www.aeu. es/aeu_webs/congreso/internet/verpostercompleto.asp?Numero=P-234

43. Oxford Centre for Evidence-Based Medicine. Levels of evidence and grades of recommendation. May 2001. Available at: http://www.cebm.net/levels_of_evidence.asp. Último acceso Diciembre del 2008 . 